\title{
Fatigue and Dyspnoea as Main Persistent Post-COVID-19 Symptoms in Previously Hospitalized Patients: Related Functional Limitations and Disability
}

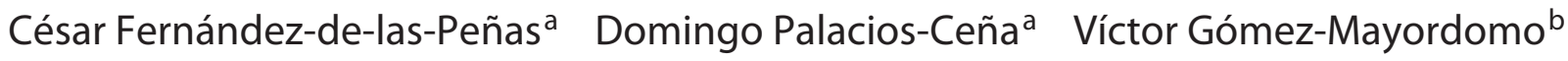 \\ María Palacios-Ceña ${ }^{a} \quad$ Jorge Rodríguez-Jiménez ${ }^{a}$ Ana l. de-la-Llave-Rincón ${ }^{a}$ \\ María Velasco-Arribasc, d Stella Fuensalida-Novo ${ }^{a}$ Silvia Ambite-Quesada ${ }^{a}$ \\ Carlos Guijarroc, d María L. Cuadrado ${ }^{b, e} \quad$ Lidiane L. Florencio $^{a} \quad$ José A. Arias-Navalón ${ }^{f}$ \\ Ricardo Ortega-Santiago $^{a}$ Carlos M. Elvira-Martínez ${ }^{b}$ Luis J. Molina-Trigueros ${ }^{a}$ g \\ Juan Torres-Macho e, h Tomas Sebastián-Vianag María Gabriela Canto-Diez $^{\text {e, h }}$ \\ Margarita Cigarán-Méndez ${ }^{\mathrm{i}}$ Valentín Hernández-Barreraj ${ }^{j}$ Lars Arendt-Nielsenk \\ aDepartment of Physical Therapy, Occupational Therapy, Physical Medicine and Rehabilitation, Universidad Rey Juan Carlos \\ (URJC), Madrid, Spain; 'beurology Department, Hospital Clínico San Carlos, Madrid, Spain; 'Department of Internal Medicine- \\ Infectious, Research Department, Hospital Universitario Fundación Alcorcón, Madrid, Spain; dDepartment of Medicine, \\ Universidad Rey Juan Carlos (URJC), Madrid, Spain; 'Department of Medicine, School of Medicine, Universidad Complutense \\ de Madrid, Madrid, Spain; ' Faculty of Health Sciences, Alfonso X el Sabio University, Madrid, Spain; ${ }^{9}$ Department of Physical

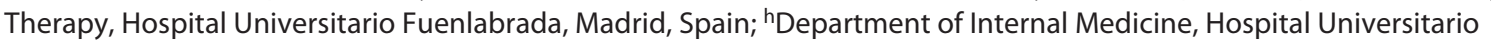 \\ Infanta Leonor-Virgen de la Torre, Madrid, Spain; 'Department of Psychology, Universidad Rey Juan Carlos (URJC), Madrid,

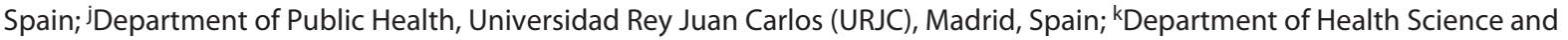 \\ Technology, Faculty of Medicine, Aalborg University, CNAP, Center for Sensory-Motor Interaction (SMI), Aalborg, Denmark
}

\section{Keywords}

COVID-19 · Fatigue · Dyspnoea $\cdot$ Function $\cdot$ Risk factors

\begin{abstract}
Background: Multicentre studies focussing on specific longterm post-COVID-19 symptoms are scarce. Objective: The aim of this study was to determine the levels of fatigue and dyspnoea, repercussions on daily life activities, and risk factors associated with fatigue or dyspnoea in COVID-19 survivors at long term after hospital discharge. Methods: Age, gender, height, weight, symptoms at hospitalization, preexisting medical comorbidity, intensive care unit admission,
\end{abstract}

and the presence of cardio-respiratory symptoms developed after severe acute respiratory syndrome coronavirus 2 infection were collected from patients who recovered from COVID-19 at 4 hospitals in Madrid (Spain) from March 1 to May 31, 2020 (first COVID-19 wave). The Functional Impairment Checklist was used for evaluating fatigue/dyspnoea levels and functional limitations. Results: A total of 1,142 patients (48\% women, age: 61, standard deviation [SD]: 17 years) were assessed 7.0 months (SD 0.6) after hospitalization. Fatigue was present in $61 \%$ patients, dyspnoea with activity in 55\%, and dyspnoea at rest in $23.5 \%$. Only 355 (31.1\%) patients did not exhibit fatigue and/or dyspnoea 7 months after hospitalization. Forty-five per cent reported functional karger@karger.com www.karger.com/res Karger
(C) 2021 S. Karger AG, Basel
Correspondence to:

César Fernández-de-las-Peñas, cesar.fernandez@urjc.es 
limitations with daily living activities. Risk factors associated with fatigue and dyspnoea included female gender, number of pre-existing comorbidities, and number of symptoms at hospitalization. The number of days at hospital was a risk factor just for dyspnoea. Conclusions: Fatigue and/or dyspnoea were present in $70 \%$ of hospitalized COVID-19 survivors 7 months after discharge. In addition, 45\% patients exhibited limitations on daily living activities. Being female, higher number of pre-existing medical comorbidities and number of symptoms at hospitalization were risk factors associated to fatigue/dyspnoea in COVID-19 survivors 7 months after hospitalization.

(c) 2021 S. Karger AG, Basel

\section{Introduction}

Evidence supports that symptoms associated with severe acute respiratory syndrome coronavirus 2 (SARSCoV-2) causing COVID-19 [1] are highly heterogeneous and range from no symptoms to minimal (cough, sneezing, or sore throat), mild (fever, rhinorrhoea, dyspnoea, or diarrhoea), or severe (pneumonia, acute respiratory distress syndrome, septic shock, or systemic multiple organ failure) symptoms [2]. The literature about COVID-19 has concentrated on the management of acute cases at hospitalization periods. However, once the acute phase has been surpassed, increasing evidence supports the presence of post-COVID-19 sequelae [3]. Since millions of people have been infected by SARS-CoV-2, it is possible that those exhibiting post-COVID-19 symptoms represent a health-care problem [4].

The number of studies investigating the presence of post-COVID-19 symptoms is small in comparison with the explosion of the literature related to the acute symptoms and management of COVID-19 [5]. Several studies have suggested that between 55 and $85 \%$ of previous hospitalized COVID-19 survivors' exhibit post-COVID-19 symptoms 2-3 months after the infection [6-12]. In fact, a recent meta-analysis published as preprint found an overall prevalence of $80 \%$ of COVID-19 survivors exhibiting at least 1 post-COVID-19 symptom, with fatigue (58\%) and dyspnoea (24\%) among the top 5 post-COVID-19 symptoms [13]. Another meta-analysis focussed on respiratory symptoms also reported that fatigue and dyspnoea are the most prevalent respiratory post-COVID-19 symptoms in hospitalized patients with a prevalence of $52 \%$ and $37 \%$, respectively, between 3 weeks and 3 months after hospital discharge [14]. However, most previous single studies [6-12] and those included in these systematic reviews $[13,14]$ did not assess the potential levels of fatigue or dyspnoea and did not evaluate the functional limitations related to these sequelae. In addition, population samples ranged from 120 to 380 participants, recruited all from a single centre, with follow-up periods of $<3$ months [6-14]. To further determine the relevance of fatigue and dyspnoea as persistent post-COVID-19 symptoms, multicentre studies including a large number of participants, follow-up periods $>6$ months after hospital discharge and potential functional limitations are needed.

There is preliminary research assessing the fatigue level and its impact on COVID-19 survivors. Jacobs et al. [13] investigated the presence of persistent symptoms at 35 days in a sample of 183 COVID-19 patients and reported that fatigue (55\%) and dyspnoea (45.3\%) were associated with worse quality of life [15]. This study classified the levels of fatigue and observed that $40 \%$ of the sample $(n=75)$ self-rated fatigue as severe [15]. In a sample of 128 COVID-19 patients, Townsend et al. [16] found that more than half $(52.3 \%)$ reported fatigue at a median follow-up period of 10 weeks after the symptom onset, which was not associated with the severity of the SARSCoV-2 infection (need for inpatient admission, supplemental oxygen, and critical care) or laboratory markers. Again, these 2 studies included small numbers of participants recruited from just 1 single centre and follow-up periods $<3$ months.

This multicentre study reports the largest systematic assessment to date of fatigue and dyspnoea as persistent post-COVID-19 symptoms in COVID-19 survivors after hospital discharge, also evaluating their repercussion on functional activity of daily living. The aims of this study were (1) to determine the levels of fatigue and dyspnoea as post-COVID-19 symptoms in COVID-19 survivors at 6 months after hospitalization; (2) to evaluate functional repercussions of these symptoms on daily living activities; and (3) to determine the potential risk factors associated with fatigue and dyspnoea in hospitalized COVID-19 survivors.

\section{Methods}

\section{Participants}

This multicentre cross-sectional cohort study was conducted in Spain from September 1 to November 30, 2020, and included hospitalized patients who had recovered from SARS-CoV-2 infection at 4 public hospitals in Madrid (Spain) during the first wave (March-May 2020). Participants should have been positively diagnosed of SARS-CoV-2 (ICD-10 code) infection with the real-time 
Table 1. Demographic and clinical data of the sample and by gender

\begin{tabular}{|c|c|c|c|}
\hline & Total sample $(n=1,142)$ & Male $(n=601)$ & Female $(n=541)$ \\
\hline Age, mean $(S D)$, years & $61(17)$ & $61.5(16)$ & $60(17.5)$ \\
\hline Gender, male/female, $n$ (\%) & $601(52.5) / 541(47.5)$ & - & - \\
\hline Weight, mean (SD), kg* & $70(15)$ & $75(13)$ & $64(14)$ \\
\hline Height, mean (SD), cm* & $166(10)$ & $171(8.5)$ & $159(7)$ \\
\hline BMI, mean (SD), $\mathrm{kg} / \mathrm{cm}^{2}$ & $25.4(3.0)$ & $25.6(4.2)$ & $25.3(2.8)$ \\
\hline \multicolumn{4}{|l|}{ Smoking status, $n(\%)^{*}$} \\
\hline Active & $96(8.5)$ & $65(10.8)$ & $31(5.7)$ \\
\hline None or former & $1,046(91.5)$ & $536(89.2)$ & $509(94.3)$ \\
\hline \multicolumn{4}{|l|}{ Medical comorbidities, $n$ (\%) } \\
\hline Hypertension* & $291(25.5)$ & $176(29.3)$ & $115(21.3)$ \\
\hline Diabetes & $145(13)$ & $90(15)$ & $55(10.2)$ \\
\hline Cardiovascular disease & $144(13)$ & $90(15)$ & $54(10)$ \\
\hline Rheumatological disease & $61(5.5)$ & $37(6.2)$ & $24(4.4)$ \\
\hline Asma & $55(5)$ & $30(5)$ & $25(4.6)$ \\
\hline Obesity & $54(5)$ & $34(5.6)$ & $20(3.7)$ \\
\hline COPD & $51(4.5)$ & $31(5.2)$ & $20(3.7)$ \\
\hline Stroke & $29(2.5)$ & $17(2.8)$ & $12(2.3)$ \\
\hline Other (cancer, kidney disease) & $105(9)$ & $52(8.6)$ & $53(9.8)$ \\
\hline Days at hospital, mean (SD)* & $14(12)$ & $14.6(12.5)$ & $12.5(11)$ \\
\hline \multicolumn{4}{|l|}{ ICU admission } \\
\hline Yes/no, $n(\%)^{*}$ & $80(7) / 1,062(93)$ & $51(8.4) / 450(91.6)$ & $29(5.4) / 512(94.6)$ \\
\hline Stay at ICU, mean (SD), days & $15(13)$ & $15(12.5)$ & $14.5(15)$ \\
\hline \multicolumn{4}{|c|}{ Cardio-respiratory post-COVID-19 symptoms, $n(\%)$} \\
\hline Fatigue* & $695(61)$ & $329(54.7)$ & $367(67.8)$ \\
\hline Dyspnoea with activity* & $627(55)$ & $289(48.1)$ & $338(62.5)$ \\
\hline Dyspnoea at rest* & $268(23.5)$ & $114(19)$ & $154(28.4)$ \\
\hline Chest pain & $80(7)$ & $42(7)$ & $38(7)$ \\
\hline Tachycardia-palpitations & $77(6.5)$ & $43(7.1)$ & $34(6.3)$ \\
\hline Cough & $24(2)$ & $12(2)$ & $12(2)$ \\
\hline
\end{tabular}

BMI, body mass index; COPD, chronic obstructive pulmonary disease; SD, standard deviation; ICU, intensive care unit. * Significant differences between males and females.

reverse transcription-polymerase chain reaction assay of nasopharyngeal/oral swab samples and the presence of consistent clinical and radiological findings at hospital admission. All eligible COVID-19 survivors discharged from the participating hospitals were included in an anonymous database, and a random selection of 300 patients from each hospital was performed with a piece of online randomization software. No exclusion criteria were applied to get the widest COVID-19 survivors' spectrum, although individuals with a documented diagnosis of dementia or delirium (or otherwise unable to conduct the interview) were excluded.

\section{Procedure}

Participants who agreed to participate were scheduled for a telephone interview by trained researchers following the procedures used in population-based survey studies. A specific questionnaire for this study was developed by a multidisciplinary research team. Clinical (e.g., age, gender, height, weight, and preexisting comorbidities) and hospitalization (days at hospital, symptoms at hospital admission, and intensive care unit [ICU] admission) data were collected from hospital medical records. Participants were systematically asked to report the presence or ab- sence of any respiratory/cardiac symptom including dyspnoea, fatigue, cough, chest pain, and palpitations developed after hospitalization and whether these symptoms persisted at the time of the study. It was emphasized that the reported symptoms should have appeared after hospital discharge. More than one symptom could be reported by the same participant.

Additionally, due to the similarities between the SARS and COVID-19, we used the items from the Functional Impairment Checklist (FIC), a disease-specific tool used for evaluating the functional consequences of SARS [17]. The FIC consists of 4 items assessing symptoms including shortness of breath (dyspnoea) at rest or with activity, fatigue, and muscle weakness. In this study, we focussed fatigue and dyspnoea symptoms; therefore, muscle weakness was excluded from the analysis. Each symptom is classified on 4 degrees of severity by the patient (0: no, 1: mild, 2 : moderate, and 3: severe). We defined mild affectation if the symptom limited $25 \%$ of the patient' activity, moderate if limited 50\%, and severe when $75 \%$ of higher. Further, the FIC also includes other 4 items assessing limitations in occupational, leisure/social activities, basic, and instrumental activities of daily life [15]. Each item is also classified on 4 degrees of severity (0: no, 1: mild, 2: moder- 
ate, and 3: severe). Mild affectation was referred when the patient perceived a $25 \%$ of limitation on the activity, moderate when the limitation was $50 \%$, and severe when perceived as $75 \%$ of higher.

\section{Statistical Analysis}

The statistical analysis was conducted with STATA 16.1 (StataCorp. 2019. Stata Statistical Software: Release 16; StataCorp LP, College Station, TX, USA). Data were presented as mean (standard deviation [SD]) or percentages as appropriate. We compared the presence of fatigue or dyspnoea levels and the related functional limitations according to gender and whether the patient had been admitted to the ICU. Bivariate analyses were conducted to determine the association between the presence of fatigue or dyspnoea and functional limitations. Multivariable unconditional logistic regression prediction and risk models were constructed to identify those variables related to clinical data (age, gender, height, weight, and number of pre-existing comorbidities), and hospitalization data (number of symptoms at hospital admission, days at hospital, and ICU/non-ICU admission) associated with the presence of persistent post-COVID-19 fatigue and dyspnoea. Adjusted odds ratio (OR) with $95 \%$ confidence intervals (95\% CIs) were calculated. A priori, the level of significance was set at 0.05 .

\section{Results}

\section{Participants}

From 4,401 patients hospitalized at the participating hospital during the first wave of the pandemic (March 1 to May 31,2020), a total of 1,200 participants were randomly selected and invited to participate. Thirteen patients refused to participate, 10 were not able to be contacted after 3 attempts, and 35 had deceased after hospital discharge. A total of 1,142 (48\% women, mean age: 61, SD: 17 years) agreed to participate and were included. The most common symptoms at hospitalization due to SARS-CoV-2 infection included fever (71\%), myalgia (35\%), dyspnoea (32\%), and cough (30\%). Four hundred and eighty-two $(n=482,42 \%)$ had no medical comorbidity, 406 (36\%) had 1 comorbidity, $174(15 \%)$ had 2 comorbidities, and the remaining 80 (7\%) had 3 comorbidities. Clinical features of the study sample and by gender are detailed within Table 1 .

Participants were assessed a mean of $7(\mathrm{SD}, 0.6)$ months after hospital discharge. In general, just 212 (18.5\%) patients were completely free of any-type of post-COVID-19 symptom, 238 (21\%) reported 1 symptom, 267 (23.5\%) had 2 symptoms, and the remaining 425 (37\%) had 3 or more.

\section{Fatigue and Dyspnoea as Long-Term Post-COVID-19 Symptoms}

Seven months after hospital discharge, 695 (61\%) patients experienced persistent fatigue, 627 (55\%) reported dyspnoea with activity, and 268 (23.5\%) dyspnoea at rest. In fact, only 355 (31.1\%) patients did not exhibit fatigue and/or dyspnoea 7 months after hospitalization. Other symptoms, less prevalent, included chest pain (7\%), tachycardia-palpitations (6.5\%), or cough (2\%) (Table 1).

Significantly higher proportions of females experienced fatigue (OR 1.75, 95\% CI: 1.37-2.24, $p<0.001$ ), dyspnoea with activity (OR 1.76, 95\% CI: $1.39-2.23, p<$ 0.001 ), and dyspnoea at rest (OR 1.70,95\% CI: 1.29-2.24), $p<0.001$ ) when compared with males (Table 1). In contrast, the presence of fatigue (OR 0.98, 95\% CI: 0.61-1.57, $p=0.963)$, dyspnoea with activity (OR 1.05, 95\% CI: 0.66 $1.93, p=0.835$ ), or dyspnoea at rest (OR $1.55,95 \% \mathrm{CI}$ : $0.94-2.55, p=0.080$ ) was not significantly different depending on ICU or not admission.

Among 695 patients reporting persistent post-COVID-19 fatigue, 353 (51\%) patients experienced it as moderate to severe, whereas the remaining 342 (49\%) perceived it as mild. Among 627 patients presenting dyspnoea with activity, $282(45 \%)$ described it as moderate to severe, and the remaining 345 (55\%) patients described it as mild. Finally, from 268 individuals presenting dyspnoea at rest, 69 (26\%) described it as moderate to severe, whereas the remaining 199 (74\%) described it as mild. Females reported mild, moderate, and severe fatigue more frequently than males ( $p<0.001$, Table 2$)$. In addition, mild, moderate, and severe dyspnoea with activity and mild and moderate, but not severe, dyspnoea at rest were also more frequent in females than in males $(p<0.001$, Table 2$)$. No significant differences in the levels of fatigue or dyspnoea (mild/moderate/severe) were observed between patients with and without ICU admission (all, $p>0.1$ ).

\section{Risk Factors Associated with Fatigue and Dyspnoea}

The regression model revealed that the presence of fatigue as a persistent post-COVID-19 symptom was significantly associated with female gender (OR 1.80, 95\% CI: $1.39-2.32, p<0.001)$, the number of medical comorbidities (OR 1.21, 95\% CI: 1.04-1.42, $p=0.012$ ), and the number of symptoms experienced at hospital admission (OR 1.55, 95\% CI: 1.34-1.80, $p<0.001$ ).

The presence of dyspnoea as persistent symptom was significantly associated with female gender (at rest: OR 1.84, 95\% CI: 1.38-2.47; activity: OR 1.86, 95\% CI: $1.45-$ $2.39 ; p<0.001$ ), number of days at hospital (rest: OR 1.02, 95\% CI: 1.01-1.03, $p<0.001$; activity: OR 1.013, 95\% CI: $1.001-1.025, p=0.025)$, number of medical comorbidities (at rest: OR 1.21, 95\% CI: 1.02-1.43, $p=0.02$; with activity: OR 1.34, 95\% CI: $1.15-1.56, p<0.001)$, and the number of symptoms at hospitalization (at rest: OR 1.21, 95\% 
Table 2. Gender differences in fatigue and dyspnoea symptoms in COVID-19 survivors

\begin{tabular}{|c|c|c|c|}
\hline & $\begin{array}{l}\text { Women }(n=541) \\
n(\%)\end{array}$ & $\begin{array}{l}\text { Men }(n=601) \\
n(\%)\end{array}$ & Adjusted OR \\
\hline \multicolumn{4}{|l|}{ Fatigue $(n=695 / 1,142)$} \\
\hline No $(n=447)$ & $174(32)$ & $273(45.5)$ & \\
\hline Mild $(n=342)$ & $169(31.5)$ & $173(28.5)$ & 1.55 (95\% Cl: 1.16-2.06), $p=0.003^{*}$ \\
\hline Moderate $(n=258)$ & $143(26.5)$ & $115(19.5)$ & 1.98 (95\% Cl: $1.44-2.69), p<0.001^{*}$ \\
\hline Severe $(n=95)$ & $55(10)$ & $40(6.5)$ & $2.12(95 \% \mathrm{Cl}: 1.36-3.32), p=0.001 *$ \\
\hline \multicolumn{4}{|c|}{ Dyspnoea on exercise $(n=627 / 1,142)$} \\
\hline No $(n=608)$ & $203(37.5)$ & $312(52)$ & \\
\hline Mild $(n=345)$ & $183(34)$ & $162(27)$ & 1.70 (95\% Cl: $1.29-2.24), p<0.001^{*}$ \\
\hline Moderate $(n=213)$ & $111(20.5)$ & $102(17)$ & 1.65 (95\% Cl: $1.20-2.28), p=0.002^{*}$ \\
\hline Severe $(n=69)$ & $44(8)$ & $25(4)$ & 2.40 (95\% Cl: $1.49-4.13), p<0.001^{*}$ \\
\hline \multicolumn{4}{|c|}{ Dyspnoea at rest $(n=268 / 1,142)$} \\
\hline No $(n=874)$ & $387(71.5)$ & $487(81.5)$ & \\
\hline Mild $(n=199)$ & $111(20.5)$ & $88(14.5)$ & 1.63 (95\% Cl: $1.19-2.22), p=0.002 *$ \\
\hline Moderate $(n=48)$ & $32(6)$ & $16(2.5)$ & $2.31(95 \%$ Cl: $1.26-4.23), p=0.007^{*}$ \\
\hline Severe $(n=21)$ & $11(2)$ & $10(1.5)$ & 1.39 (95\% Cl: 0.58-3.31), $p=0.454$ \\
\hline
\end{tabular}

OR, odds ratio; $\mathrm{Cl}$, confidence interval. * Significant differences between males and females.

Table 3. Gender differences in limitations in occupational, leisure/social activities, basic, or instrumental activities of daily life

\begin{tabular}{|c|c|c|c|}
\hline & $\begin{array}{l}\text { Women }(n=541) \text {, } \\
n(\%)\end{array}$ & $\begin{array}{l}\text { Men }(n=601), \\
n(\%)\end{array}$ & Adjusted OR \\
\hline \multicolumn{4}{|c|}{ Limitation in occupational activities ( $n=258 / 1,142)$} \\
\hline No $(n=884)$ & $397(73.5)$ & $487(81.5)$ & \\
\hline Mild $(n=134)$ & $68(12.5)$ & $66(11)$ & 1.24 (95\% Cl: 0.85-1.78), $p=0.252$ \\
\hline Moderate $(n=68)$ & $42(8)$ & $26(4)$ & 1.92 (95\% Cl: $1.15-3.20), p=0.012^{*}$ \\
\hline Severe $(n=56)$ & $34(6)$ & $22(3.5)$ & 1.88 (95\% Cl: $1.08-3.27), p=0.025^{*}$ \\
\hline \multicolumn{4}{|c|}{ Limitation in leisure/social activities ( $n=369 / 1,142)$} \\
\hline No $(n=773)$ & $331(61.5)$ & $442(74)$ & \\
\hline Mild $(n=223)$ & $117(21.5)$ & $106(17.5)$ & 1.41 (95\% Cl: 1.04-1.90), $p=0.024^{*}$ \\
\hline Moderate $(n=106)$ & $66(12)$ & $40(6.5)$ & 2.18 (95\% Cl: $1.44-3.32), p<0.001^{*}$ \\
\hline Severe $(n=40)$ & $27(5)$ & $13(2)$ & 2.75 (95\% Cl: $1.40-5.41), p=0.003^{*}$ \\
\hline \multicolumn{4}{|c|}{ Limitation in basic activities of daily life $(n=217 / 1,142)$} \\
\hline No $(n=925)$ & $424(78.5)$ & $501(83.5)$ & \\
\hline Mild $(n=132)$ & $64(12)$ & $68(11)$ & $1.13(95 \%$ Cl: 0.78-1.63), $p=0.501$ \\
\hline Moderate $(n=52)$ & $33(6)$ & $19(3)$ & 2.06 (95\% Cl: 1.15-3.68), $p=0.014^{*}$ \\
\hline Severe $(n=33)$ & $20(3.5)$ & $13(2.5)$ & $1.82(95 \%$ Cl: $0.89-3.71), p=0.096$ \\
\hline \multicolumn{4}{|c|}{ Limitation in instrumental activities of daily life $(n=309 / 1,142)$} \\
\hline No $(n=833)$ & $356(66)$ & $477(79.5)$ & \\
\hline Mild $(n=181)$ & $101(18.5)$ & $80(13)$ & 1.71 (95\% Cl: 1.24-2.37), $p=0.001^{*}$ \\
\hline Moderate $(n=90)$ & $61(11.5)$ & $29(5)$ & 2.96 (95\% Cl: 1.86-4.73), $p<0.001^{*}$ \\
\hline Severe $(n=38)$ & $23(4)$ & $15(2.5)$ & 1.65 (95\% Cl: 0.86-3.18), $p=0.131$ \\
\hline
\end{tabular}

CI: $1.02-1.45, p=0.03$; activity: OR $1.37,95 \%$ CI: $1.18-$ $1.58, p<0.001$ ). No particular medical comorbidity (e.g., hypertension, diabetes, and asthma) was associated as a risk factor for suffering from fatigue and dyspnoea as persistent post-COVID-19 symptoms.

\section{Functional Limitations and Disability}

Forty-five per cent ( $n=508,55 \%$ female) of COVID-19 survivors experienced at least 1 functional limitation with daily living activities. Two hundred and fifty-eight $(n=$ $258,22.5 \%)$ patients experienced limitations with previous 
Fig. 1. Prevalence of fatigue, dyspnoea at rest, dyspnoea with activity, and functional limitations with occupational, social/leisure and instrumental, and basic daily living activities in female and male previously hospitalized COVID-19 survivors.

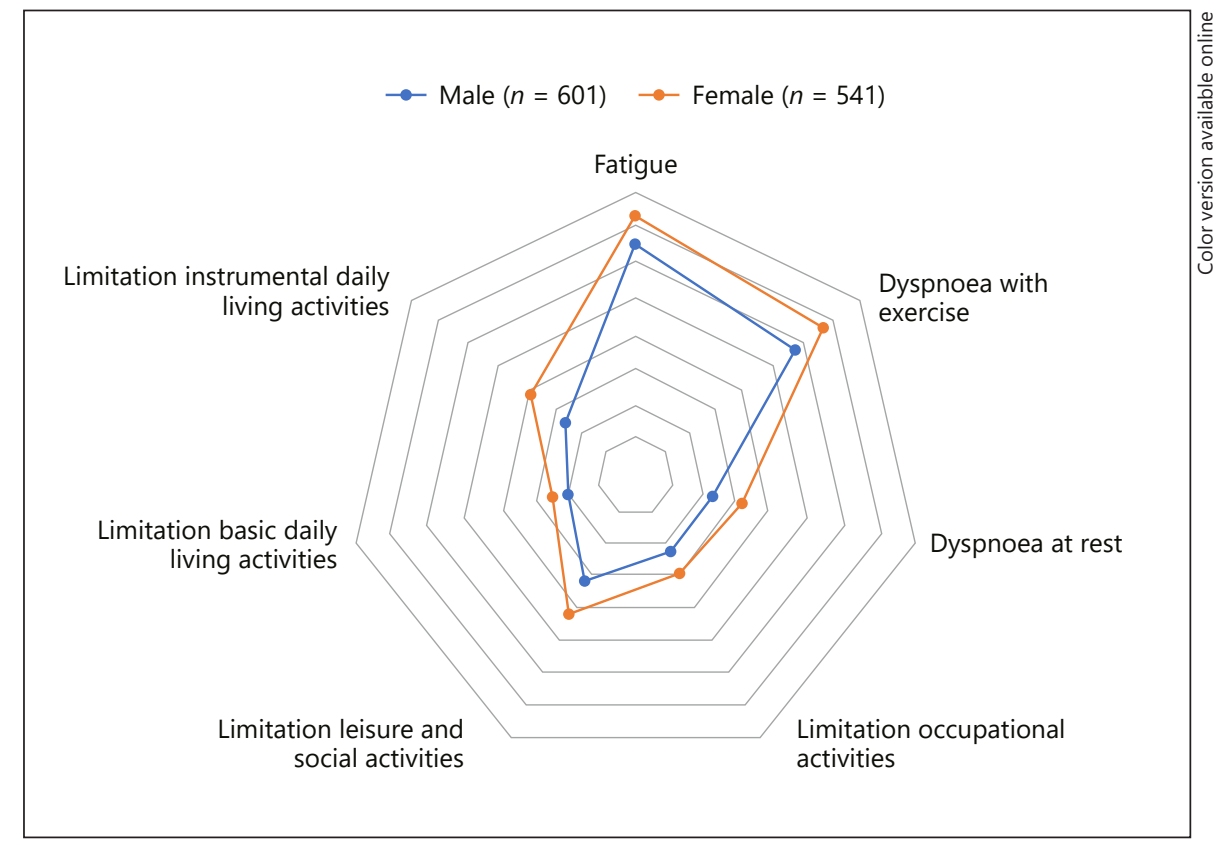

occupational activities, 369 (32\%) with social/leisure activities, 309 (27\%) with instrumental activities, and 217 (19\%) with basic activities of daily living. In general, a greater proportion of females (OR 1.75, 95\% CI: 1.38-2.22, $p<0.001)$ experienced limitations with daily living activity than males. In particular, higher proportions of females showed limitations with occupational activities (144/541, $26 \%$ OR 1.50, 95\% CI: 1.14-1.98, $p=0.004)$, leisure/social activities (210/541, 39\%, OR 1.72, 95\% CI: 1.34-2.21, $p<$ $0.001)$, instrumental daily living activities (185/541, 34\%, OR 1.99, 95\% CI: $1.53-2.60, p<0.001$ ), and basic activities of daily living (117/541, $21.5 \%$, OR 1.41, 95\% CI: 1.05-2.60, $p=0.022$ ) compared to males (occupational activities: 114/601, 19\%; social/leisure activities: 159/601, 26.5\%; instrumental activities of daily living: 124/601, 20.5\%; and basic activities of daily living: 100/601, 16.5\%).

Within those participants showing daily living activity limitations, $48 \%(n=124 / 258)$ had moderate to severe limitations in occupational activities, $39.5 \%(n=146 / 369)$ showed moderate to severe limitations in leisure/social activities and $41.5 \%(n=128 / 309)$, and $39 \%(n=85 / 217)$ moderate/severe limitations for instrumental and basic daily living activities, respectively. Females reported moderate to severe limitations in occupational activities, any limitation in leisure/social activity, mild/moderate limitations in instrumental activity of daily living, and moderate limitations in basic activities of daily living more frequently than males (Table 3 ). Figure 1 graphs the differences between female and male patients.
Additionally, significantly higher proportions of patients requiring ICU admission reported limitations with occupational activities (OR 2.16, 95\% CI: 1.34-3.48, $p=$ 0.001), leisure/social activities (OR 1.98, 95\% CI: $1.26-$ $3.14, p=0.003$ ), instrumental daily living activities (OR 2.34, 95\% CI: $1.48-3.72, p<0.001)$, and basic activities of daily living (OR 2.95, 95\% CI: 1.83-4.75, $p<0.001$ ) when compared to those not requiring ICU admission. In fact, a greater proportion of patients receiving ICU admission experienced moderate and severe, but not mild, limitations with daily living activities (occupational: moderate OR 2.57, 95\% CI: 1.20-5.49, $p=0.015$-severe OR 5.13, 95\% CI: 2.59-10.17, $p<0.001$; leisure/social: moderate OR 2.89, 95\% CI: $1.54-5.42, p=0.001$-severe OR 4.33, 95\% CI: 1.88-9.98, $p<0.001$; instrumental: moderate OR 3.91, 95\% CI: 2.10-7.27, $p<0.001-$ severe OR 6.37, 95\% CI: 2.91-13.94, $p<0.001$; and basic: moderate OR 3.72, 95\% CI: $1.72-8.07, p=0.001-$ severe OR 7.74, 95\% CI: $3.49-17.15, p<0.001)$ than those not requiring ICU admission.

Significant positive associations between the presence of fatigue/dyspnoea (either at rest or with activity) and limitations on daily living activities $(r$ : 0.359-0.684, all $p<0.001$ ) were found: those patients with higher levels of fatigue or dyspnoea exhibited more severe limitations on daily living activities. No significant associations between the presence of pre-existing comorbidities and functional limitations were seen (all, $p>0.20$ ). 


\section{Discussion}

This study describes the incidence of persistent fatigue and dyspnoea in the largest multicenter study published to date and with a long-term follow-up period in hospitalized COVID-19 survivors. The results revealed that $70 \%$ of hospitalized COVID-19 survivors exhibited fatigue and/or dyspnoea 7 months after hospitalization. Further, $45 \%$ also reported at least 1 limitation on their daily living activities. Female gender, number of pre-existing comorbidities, and the number of symptoms at hospital admission were risk factors potentially associated with the presence of fatigue or dyspnoea 7 months after hospital discharge, whereas the number of days at hospital was just associated with the presence of dyspnoea.

The presence fatigue or dyspnoea in previously hospitalized COVID-19 survivors after hospitalization is consistent with current data, which also reported a high prevalence of these symptoms but at shorter follow-up periods (2-3 months after) [6-16]. Our study confirms that fatigue and dyspnoea continue being highly prevalent at long-term after hospital discharge supporting that they can be persistent post-COVID-19 symptoms. In fact, the presence of persistent respiratory sequelae after the SARS-CoV-2 infection is similar to previous data found in patients who had suffered from SARS or Middle East respiratory syndrome, who also experienced reduced lung function and function capacity 6 months after infection [18]. In fact, the presence of persistent fatigue agrees with the hypothesis of a post-viral fatigue syndrome associated to coronavirus infection [19].

Nevertheless, discrepancies are observed in the presence of fatigue in hospitalized patients after SARS-CoV-2 since a Chinese study has reported that $86 \%$ of hospitalized patients were symptom-free 1 month after hospital discharge [20]. Similarly, data from non-hospitalized patients are also conflicting since Stavem et al. [21] observed that only $16 \%$ of non-hospitalized patients had dyspnoea between 2 and 6 months after symptom onset, whereas Goërtz et al. [22] found that $90 \%$ of non-hospitalized COVID-19 survivors experienced fatigue and/or dyspnoea 3 months after infection. Different reasons including gender, population age, medical comorbidities, racial disparities [23], or blood type [24] could explain these discrepancies.

In addition to the presence or absence of fatigue and dyspnoea, it is also important to determine the level of severity and functional impairment. Around 50\% of hospitalized patients self-rated their fatigue as moderate/severe, in agreement with data reported by Jacobs et al. [13].
Moreover, we differentiated the presence of dyspnoea at rest and with activity, a distinction that has not been specified in the former post-COVID-19 literature. Our results showed that $50 \%$ of patients reported dyspnoea with activity, but $25 \%$ experienced dyspnoea at rest. Differentiation between these 2 situations could be clinically relevant since the presence of dyspnoea at rest could represent more severe respiratory sequelae associated with lung damage than the presence of dyspnoea with activity. This distinction would be in line with the fact that $25 \%$ of hospitalized COVID-19 survivors experienced functional limitations during daily living activities but at different levels, for example, social, occupational, instrumental, or basic. We also observed that limitations during daily living activities were associated to the presence of fatigue and dyspnoea. Current data are similar to those previously observed by Jacobs et al. [13] who also reported that fatigue and dyspnoea were associated with worse quality of life and more difficulty during different daily living activities, for example, walking, climbing stairs, or lifting. Additionally, these authors did not find an association between any particular pre-existing comorbidity and post-COVID-19 quality of life, suggesting that functional limitations seem to be specifically related to COVID-19 [13]. Our results support this assumption since we also did not observe an association between the number of pre-existing comorbidities and functional limitations.

Current findings support an impact of persistent postCOVID-19 respiratory symptoms on function in previously hospitalized COVID-19 patients. Future studies should identify which particular daily living activities are more impaired to better determine appropriate therapeutic physical activity strategies. Determining the exact mechanisms of post-COVID-19 fatigue and dyspnoea is beyond the scope of this study, but some hypotheses related to current findings will be discussed.

It has been hypothesized that prolonged pro-inflammatory response (hyper-inflammatory cytokine storm) associated with SARS-CoV-2 infection may root an atypical response of the mast cells, promoting the development of the plethora of post-COVID symptoms [25]. A second hypothesis would be related to the presence of persistent viral replication after the acute phase [26]. A third hypothesis includes the presence of residual lung injury. In line with this hypothesis, a recent review has reported that almost $50 \%$ of the patients with COVID-19 present residual abnormalities on the lungs almost 3 months after hospital discharge [27]. In fact, the presence of residual injury in the lungs could explain the differences in the prevalence of dyspnoea with exercise (55\%), 
which could be related to better functional status, when compared to the presence of dyspnoea at rest (23.5\%). In addition, the presence of persistent post-COVID-19 respiratory symptoms in subgroups of patients and the differences in functional repercussion on daily living activities would support that different mechanisms are involved at the same time in post-COVID-19.

This study also identified some potential risk factors associated with persistent post-COVID-19 fatigue and dyspnoea. Female gender, higher number of comorbidities, and higher number of symptoms at the acute phase of the infection were risk factors associated with persistent fatigue and dyspnoea 7 months after hospital discharge. These results agree with those previously reported by Jacobs et al. [13] and Townsend et al. [16] who also showed higher prevalence of fatigue in female patients. Interestingly, the prevalence of COVID-19 is similar in males and females, but males exhibit greater severity [28, 29]. These results would suggest gender differences in the manifestations of COVID-19, with men exhibiting worse manifestations during the acute phase but women presenting more persistent symptoms in the long-term. Future studies are now needed to investigate the possible mechanisms underlying these differences.

In agreement with our results, Goërtz et al. [22] likewise showed that pre-existing comorbidities and the number of symptoms at the onset of infection were associated with the number of persistent symptoms in nonhospitalized patients; however, these authors did not specify the presence of fatigue on these associations. We also observed that the number of pre-existing comorbidities and the number of symptoms at infection onset were associated with the presence of fatigue and dyspnoea in hospitalized patients. As it can be observed in Table 1, hypertension and diabetes were the medical comorbidities more prevalent in our sample. However, no specific comorbidity was associated with fatigue or dyspnoea in our multivariate analysis. In agreement with our results, recent studies suggest that the presence of respiratory comorbidities, such as asthma, is not associated with the presence of more post-COVID-19 symptoms [30, 31]. It is possible that SARS-CoV-2 infection can promote persistent post-COVID-19 symptoms, for example, fatigue, in predisposing individuals with higher number of preexisting medical comorbidities. In such a scenario, more medical comorbidities and the presenting more symptoms during the acute phase of the infection could precipitate a cascade of events in these individuals. It is interesting to note that fatigue has not been found to be associated with infection severity or laboratory markers, suggesting the absence of a specific immune signature associated with this post-COVID-19 fatigue [16]. Future studies should investigate these hypotheses.

These results should be considered according to its strengths and limitations. Among strengths, firstly, this is a large multicentre study and with a long-term follow-up period to date investigating persistent fatigue and dyspnoea as post-COVID-19 symptoms. Secondly, we used a specific-disease questionnaire for evaluating fatigue, dyspnoea, and limitations on daily activities; although, we should recognize that this questionnaire has been $s$ validated for SARS but not for COVID-19. Among the limitations, we only included hospitalized patients; therefore, we cannot extrapolate the current results to nonhospitalized patients. Thirdly, the number of patients requiring ICU admission was small, so their comparisons should be considered with caution at this stage. Fourthly, we used a cross-sectional design; therefore, we do not have data about the evolution of these symptoms with time. Fifthly, we included Caucasian, not African or Asian, participants; therefore, extrapolation of our results to different ethnicities or races should be not performed. Finally, we collected self-reported patient outcomes, but not objective measures, for example, blood oxygen saturation, inflammatory biomarkers, or chest X-ray, which could help to further define pulmonary impairments in future studies.

\section{Conclusions}

This study found that almost $70 \%$ of previously hospitalized COVID-19 survivors experience fatigue and/or dyspnoea 7 months after hospitalization. Further, 45\% also showed any limitation on daily living activity. Female gender, the number of pre-existing comorbidities, and the number of symptoms at the acute phase of the infection at hospital admission were risk factors potentially associated with fatigue and dyspnoea, whereas the number of days at hospital was associated with the presence of dyspnoea. Identification and recognition of post-COVID-19 symptoms will ensure immediate action and counselling for patients who may otherwise struggle with unrecognized and unmanaged symptoms.

\section{Acknowledgements}

The Center for Neuroplasticity and Pain (CNAP) is supported by the Danish National Research Foundation (DNRF121). 


\section{Statement of Ethics}

The study was conducted following the Helsinki Declaration and approved by the Local Ethics Committees of all the institutions involved (HCSC20/495E, HUFA 20/126, HUIL/092-20, HUF/EC1517, URJC0907202015920). Participants provided their verbal informed consent accepting their participation before collecting data telephonically. The verbal consent was considered appropriate by the Ethics Committee of all institutions involved (Hospital Clínico San Carlos, Hospital Universitario Fundación Alcorcón, Hospital Universitario Infanta Leonor, Hospital Universitario Fuenlabrada, Universidad Rey Juan Carlos) due to the pandemic situation.

\section{Conflict of Interest Statement}

No conflict of interest is declared by any of the authors.

\section{Funding Sources}

No funds were received for this study.

\section{Author Contributions}

All authors contributed to the study concept and design. C.F.P., D.P.-C., V.G.-M., and V.H.-B. conducted literature review and did the statistical analysis. V.G.-M, M.V.-A., C.G., C.M.E.-M., M.L.C., J.A.A.-N., L.J.M.-T., T.S.-V., J.T.-M., and M.G.C.-D. recruited participants. J.R.-J., M.P.-C., A.I.L.-R., S.F.-N., L.L.F, R.O.S., M.C.-M., and S.A.-Q. collected data. L.A.-N. supervised the study. All the authors contributed to interpretation of data. C.F.P., D.P.C., V.G.-M., M.L.C., and L.A.-N. contributed to drafting the manuscript. All the authors revised the text for intellectual content and have read and approved the final version of the manuscript.

\section{Data Availability Statement}

All data generated or analysed during this study are included in this article. Further enquiries can be directed to the corresponding author.

\section{References}

1 Zhu N, Zhang D, Wang W, Li X, Yang B, Song $\mathrm{J}$, et al. A novel coronavirus from patients with pneumonia in China, 2019. N Engl J Med. 2020;382:727-33.

2 Guan WJ, Ni ZY, Hu Y, Liang WH, Ou CQ, $\mathrm{He} \mathrm{JX}$, et al. Clinical characteristics of coronavirus disease 2019 in China. N Engl J Med. 2020;382:1708-20.

3 Marshall M. The lasting misery of coronavirus long-haulers. Nature. 2020;585:339-41.

4 Rubin R. As their numbers grow, COVID-19 "long haulers" stump experts. JAMA. 2020; 324:1381-3.

5 Mahase E. COVID-19: what do we know about "long COVID"? BMJ. 2020;370:m2815.

6 Carfi A, Bernabei R, Landi F; Gemelli Against COVID-19 Post-Acute Care Study Group. Persistent symptoms in patients after acute COVID-19. JAMA. 2020;324(6):603-5.

7 Garrigues E, Janvier P, Kherabi Y, Le Bot A, Hamon A, Gouze H, et al. Post-discharge persistent symptoms and health-related quality of life after hospitalization for COVID-19. J Infect. 2020;81:e4-6.

8 Carvalho-Schneider C, Laurent E, Lemaignen A, Beaufils E, Bourbao-Tournois C, Laribi S, et al. Follow-up of adults with noncritical COVID-19 two months after symptom onset. Clin Microbiol Infect. 2021;27(2):258-63.

9 Halpin SJ, McIvor C, Whyatt G, Adams A, Harvey O, McLean L, et al. Post-discharge symptoms and rehabilitation needs in survivors of COVID-19 infection: a cross-sectional evaluation. J Med Virol. 2021;93(2):1013-22.

10 Arnold DT, Hamilton FW, Milne A, Morley AJ, Viner J, Attwood M, et al. Patient outcomes after hospitalisation with COVID-19 and implications for follow-up: results from a prospective UK cohort. Thorax. 2020;76(4): 399-401.

11 Mandal S, Barnett J, Brill SE, Brown JS, Denneny EK, Hare SS, et al. "Long-COVID": a Cross-Sectional Study of persisting symptoms, biomarker and imaging abnormalities following hospitalisation for COVID-19. Thorax. 2021 Apr;76(4):396-8.

12 Nehme M, Braillard O, Alcoba G, Aebischer Perone S, Courvoisier D, Chappuis F, et al. COVID-19 symptoms: longitudinal evolution and persistence in outpatient settings. Ann Intern Med. 2021;174(5):723-5.

13 Jacobs LG, Gourna Paleoudis E, Lesky-Di Bari D, Nyirenda T, Friedman T, Gupta A, et al. Persistence of symptoms and quality of life at 35 days after hospitalization for COVID-19 infection. PLoS One. 2020;15:e243882.

14 Lopez-Leon S, Wegman-Ostrosky T, Perelman C, Sepulveda R, Rebolledo PA, Cuapio A, et al. More than 50 long-term effects of COVID-19: a systematic review and meta-analysis. Sci Rep. 2021 Aug 9;11(1):16144.

15 Cares-Marambio K, Montenegro-Jiménez Y, Torres-Castro R, Vera-Uribe R, Torralba Y, Alsina-Restoy X, et al. Prevalence of potential respiratory symptoms in survivors of hospital admission after coronavirus disease 2019 (COVID-19): a systematic review and metaanalysis. Chron Respir Dis. 2021 Jan-Dec;18: 14799731211002240.

16 Townsend L, Dyer AH, Jones K, Dunne J, Mooney A, Gaffney F, et al. Persistent fatigue following SARS-CoV-2 infection is common and independent of severity of initial infection. PLoS One. 2020;15:e240784.
17 Lam SP, Tsui E, Chan KS, Lam CL, So HP. The validity and reliability of the functional impairment checklist (FIC) in the evaluation of functional consequences of severe acute respiratory distress syndrome (SARS). Qual Life Res. 2006;15:217-31.

18 Ahmed H, Patel K, Greenwood DC, Halpin S, Lewthwaite P, Salawu A, et al. Long-term clinical outcomes in survivors of severe acute respiratory syndrome (SARS) and middle east respiratory syndrome coronavirus (MERS) outbreaks after hospitalisation or ICU admission: a systematic review and meta-analysis. J Rehabil Med. 2020;52(5):jmr00063.

19 Wilson C. Concern coronavirus may trigger post-viral fatigue syndromes. New Sci. 2020; 246:10.

20 Wang $\mathrm{X}$, Xu H, Jiang $\mathrm{H}$, Wang L, Lu C, Wei $\mathrm{X}$, et al. The clinical features and outcomes of discharged coronavirus disease 2019 patients: a prospective Cohort Study. QJM. 2020; 113(9):657-65.

21 Stavem K, Ghanima W, Olsen MK, Gilboe HM, Einvik G. Persistent symptoms 1.5-6 months after COVID-19 in non-hospitalised subjects: a population-based Cohort Study. Thorax. 2020;76:405-7.

22 Goërtz YMJ, Van Herck M, Delbressine JM, Vaes AW, Meys R, Machado FVC, et al. Persistent symptoms 3 months after a SARSCoV-2 infection: the post-COVID-19 syndrome? ERJ Open Res. 2020;6:00542-2020.

23 Mackey K, Ayers CK, Kondo KK, Saha S, Advani SM, Young S, et al. Racial and ethnic disparities in COVID-19-related infections, hospitalizations, and deaths: a systematic review. Ann Intern Med. 2021;174(3):362-73. 
$24 \mathrm{Wu}$ BB, Gu DZ, Yu JN, Yang J, Shen WQ. Association between $\mathrm{ABO}$ blood groups and COVID-19 infection, severity and demise: a systematic review and meta-analysis. Infect Genet Evol. 2020;84:104485.

25 Afrin LB, Weinstock LB, Molderings GJ. Covid-19 hyperinflammation and post-Covid-19 illness may be rooted in mast cell activation syndrome. Int J Infect Dis. 2020;100:32732.

26 Jacobs JJL. Persistent SARS-2 infections contribute to long COVID-19. Med Hypotheses. 2021;149:110538
27 So M, Kabata H, Fukunaga K, Takagi H, Kuno $\mathrm{T}$. Radiological and functional lung sequelae of COVID-19: a systematic review and metaanalysis. BMC Pulm Med. 2021;21(1):97.

28 Jin JM, Bai P, He W, Wu F, Liu XF, Han DM, et al. Gender differences in patients with $\mathrm{CO}$ VID-19: Focus on severity and mortality. Front Public Health. 2020;8:152.

29 Gebhard C, Regitz-Zagrosek V, Neuhauser HK, Morgan R, Klein SL. Impact of sex and gender on COVID-19 outcomes in Europe. Biol Sex Differ. 2020;11:29.
30 Garcia-Pachon E, Grau-Delgado J, SolerSempere MJ, Zamora-Molina L, Baeza-Martinez C, Ruiz-Alcaraz S, et al. Low prevalence of post-COVID-19 syndrome in patients with asthma. J Infect. 2021 Jun;82(6):276316

31 Fernández-de-las-Peñas C, Torres-Macho J, Velasco-Arribas M, Arias-Navalón JA, Guijarro C, Hernández-Barrera V, et al. Similar prevalence of long-term post-COVID symptoms in patients with asthma: A case-Control Study. J Infect. 2021 Aug;83(2):237-79. 